\title{
Interação mãe-criança: enfoques teóricos e implicações decorrentes de estudos empíricos
}

\author{
Maria Aparecida Trevisan Zamberlan \\ Universidade Estadual de Londrina
}

\begin{abstract}
Resumo
Este trabalho consistiu na revisão das principais posições teóricas sobre a relação mãe-criança e as decorrências metodológicas de tais concepções nos dados empíricos delas resultantes. A análise desses dados permite destacar dois aspectos: (1) que a presença da mãe age como facilitador para um conjunto significativo de aspectos do desenvolvimento do bebê, com destaque para a organização de "estados", características sensório-perceptivas, cognição a partir do elo de ligação criado entre ambos; (2) muitas características dos infantes importantes à interação eliciam responsividade e cuidados dos agentes com os quais os bebês interagem no sentido de harmonizar-se ou de sincronizar-se em tal relação. Essa mutualidade já se faz presente nos primórdios do desenvolvimento social inicial.
\end{abstract}

Palavras-chave: Interação mãe-criança, Enfoques teórico-metodológicos, Desenvolvimento infantil, Relações, Características maternas e do infante.

\begin{abstract}
Mother-child relationship: Theoretical views and implications of empirical data review.. This paper consisted of a review of the most important theoretical views about the mother child relationship on the conceptual approaches and their possible effects on methodology. Empirical data resulted from research on the area have shown that: (1) the mother is an effective facilitator on a set of domains of infant's development, with focus on the "states organization", sensorial-perceptual capacities and cognition; (2) characteristics of infant and mother as interactive agents - mutuality - aiming to promote harmonic and synchronic relationship between the dyad are present on early social development.
\end{abstract}

Key words: Mother-child interaction, Theoretical methodological approaches, Child's development, Relationships, Mothers and infant's characteristics

$\mathrm{D}$ iferentes visões têm fundamentado teoricamente as pesquisas conduzidas sobre a interação mãe-criança. Essa relação tem sido interpretada sob diferentes perspectivas teórico- conceituais, dentre elas, destacandose o enfoque do desenvolvimento biológico evolucionário (Ainsworth \& Wittig, 1969; Blurton Jones, 1983; Bowlby, 1951/ 1991, 1989); a perspectiva motivacional associada a drives e relações objetais (Horner, 1988); a perspectiva da aprendizagem social (Baumrind \& Black, 1967), a qual salienta, particularmente, o processo de imitação como padrão de influências na socialização; a perspectiva sócio-cognitivista, que enfatiza as assimilações e co-regulações como funções abrangidas no sistema de trocas entre parceiros (Fogel, 1988; 1992; 1994; Kochanska, 1997; Schafer, 1996) e a perspectiva analíticofuncional, de base comportamental (Skinner, citado por Stedman, 1991).

A importância dessas contribuições acerca da relação infante - mãe tem sido reconhecida sob vários de seus aspec- tos: servindo como eixo de referência a partir do qual se analisam demandas ambientais adaptadas às várias etapas do desenvolvimento da criança (Biasoli-Alves, 1992; Horner,1988); como organizador da vida psíquica da criança, por possibilitar identificações que poderão influenciar seu desenvolvimento posterior (Oppenhein, Sagi \& Lamb, 1988) ou, ainda, servindo como um mediador de vários tipos de experiências cognitivas e de socialização à criança (Feinman \& Lewis, 1988; Power \& Chapiesky, 1986; Pratt, Cowan, Kerig \& Cowan, 1988).

\section{Diversidade de orientação teórica}

O estudo da interação mãe-criança tem sido amplamente desenvolvido nas últimas quatro décadas, a partir do trabalho de Bowlby (1951/1991) sobre a conduta do apego. A visão evolucionária do apego propõe que os infantes da maioria das espécies animais nascem pré-programados para o es- 
tabelecimento de uma ligação próxima e forte ao agente principal de cuidados com o qual tal relação se estabelece.

Sob um ponto de vista evolucionário, postula-se que o crescimento cerebral é intenso e extremamente rápido, até os 18 meses, bem como que o cérebro, neste período, é dotado de maior plasticidade. Isto sugere que a interação inicial com diferentes tipos de padrões de estímulos seja muito relevante. O código genético pré-seleciona determinadas características do infante que o predispõem à ligação com um agente humano adulto, com o qual desenvolve laços de apego e proteção. Segundo Ainsworth (Ainsworth \& Wittig, 1969), o apego é uma ligação afetiva que forma laços temporais e espaciais. Os comportamentos indicativos de apego buscam obter ou manter proximidade, interação e comunicação, mesmo que à distância. Neles, estão incluídos os comportamentos de aproximar-se, seguir, sorrir, chorar e chamar, quando a criança é separada de um agente específico.

A base do apego como um fator primário para o estabelecimento de relações configura-se, prontamente, na vinculação inicial mãe- infante. Em decorrência disso tal questão tem sido analisada concomitantemente com a relação mãe- criança.

Os estudos mais recentes do enfoque etológico mostram que essa relação vincular é produto da ativação de sistemas comportamentais tanto da mãe como da criança, dentro de um processo de bidirecionalidade (Schaffer, 1996, enfatizando Ainsworth et. al., 1978).

A teoria do apego sugere que a forma de relações seguras com o agente adulto é importante para a criança desenvolver sistemas comportamentais flexíveis, de modo a adaptar-se facilmente a novas situações, incluindo demandas de contato com locais e outros adultos estranhos (RossettiFerreira, 1986). O estresse da separação da mãe pode, assim, ser minimizado quando os infantes desenvolvem uma condição de apego seguro.

A relação positiva mãe-criança tem sido associada à segurança do apego no primeiro ano de vida, tanto quanto à menor reincidência de problemas sócio-emocionais e cognitivos nos anos pré-escolares.

A interpretação psicodinâmica postula que a relação emocional mãe- criança se realizaria concorrentemente ao desenvolvimento libidinal - estabelecimento da relação objetal - na história inicial da evolução do ego (Freud, 1959). Ampliando essa visão, Horner (1988) propõe uma dimensão afetivo-cognitiva que tem lugar nas interações que o infante estabelece com a mãe e que se desenvolve ao longo de toda a infância, iniciando-se pela satisfação de necessidades básicas, seguidas da necessidade de independência, auto-confiança e realização.

Bowlby (1951/1991) propôs uma interpretação substitutiva dos princípios psicanalíticos relacionados à natureza e formação dos vínculos infantis, considerando-os primários ou instintivos na espécie, e não uma aquisição secundária associada às necessidades fisiológicas da criança.

Horowitz (1987) propôs um modelo estrutural-funcional de desenvolvimento que sugere a reaproximação de características organísmicas do infante e de aspectos ambientais, considerando grupos distintos de comportamentos: os universais - que constituem-se de características pré-selecionadas na espécie e que respondem por continuidades no desenvolvimento e os não universais - relativos às aquisições mediadas pela sociedade e cultura.

Dentro da vertente comportamental, a teoria da Aprendizagem Social enfatiza a importância da associação da presença materna considerada um poderoso reforçador secundário, face a suas associações repetidas com reforçadores primários, como a alimentação e a redução do desconforto ao infante.

Do ponto de vista comportamental funcionalista, Skinner (citado por Stedman, 1991) sugeriu que pais, assim como infantes, dispõem de certas conseqüências que se ajustam ao controle de ambos os comportamentos e que tal se dá no contexto de uma interação diádica.

$\mathrm{Na}$ perspectiva das diversas abordagens revistas fica explícito que o conjunto de comportamentos que unem uma pessoa a outra ou a outro objeto, formando-se importante ligação entre elas pode ser entendido como apego. Concluise que a responsividade da mãe para com os cuidados ao bebê direciona seus sistemas comportamentais e de apego em relação a esse agente.

\section{O sistema de interação mãe-bebê}

Na maioria dos estudos conduzidos nas áreas do desenvolvimento inicial e de relações sociais, a interação entre mãe e criança tem sido considerada um recorte importante para o estudo da organização comportamental e competências do bebê, adquiridas no contexto dessa relação.

Desde a concepção, mães e bebês participam de um sistema muito complexo de relações, o qual emerge, se organiza e se modifica através do curso da evolução e de eventos culturais interpostos ao desenvolvimento subseqüente de ambos. Durante os primeiros anos de vida, mudanças significativas ocorrem tanto nas condições do infante como nas da mãe, afetando-se, mutuamente, dentro de um padrão interacional singular. A qualidade da interação inicial é considerada um importante fator mediador entre os eventos perinatais e o seu posterior desenvolvimento, particularmente no que se referem à comunicação, socialização e cognição

A competência social no sistema é definida por um construto, que se caracteriza operacionalmente pela habilidade para perceber e responder adequadamente às necessidades e formas comunicativas da criança, assumindo, portanto, uma posição central nos eventos que marcam as experiências iniciais dessa díade.

Por causa do interesse e relevância social dessa relação à prática de intervenção psico-educacional e clínica, um objetivo importante nessa área, seria o de identificar as condições estabelecedoras dessa interação que promovem características infantis e maternas estáveis.

Muitos dos componentes específicos dessa relação foram estudados nas três últimas décadas e alguns resultados de pesquisa podem ser de grande interesse à aplicação nos diversos contextos em que essa relação se evidencia. Assim, supõe-se que uma revisão geral de dados de pesquisas sobre 
os vários fatores que afetam tal relação pode trazer importantes contribuições à prática de intervenção.

\section{Revisão de estudos empíricos}

Tem sido evidenciado que a criança, desde o nascimento, possui uma estrutura capaz de responder a estímulos sociais e organização comportamental para iniciar e continuar uma interação social.

Osofsky e Connors (1979), numa revisão clássica do sistema mãe -criança, analisaram uma série de fatores maternos e infantis, como expectativas, experiência e atitudes maternas e tipos de características, estilos e disposições dos infantes que desempenham um papel significativo no desenvolvimento dessas relações. Esse conhecimento produziu mudanças, tanto nas práticas de cuidados diários de mães em relação a suas crianças quanto nas rotinas hospitalares e de cuidados institucionais de bebês em berçários e creches.

Os resultados de trabalhos conduzidos na área, relacionando características maternas e o desenvolvimento presente e ulterior da criança apontam elos entre sensibilidade materna, qualidade da ligação estabelecida e correlatos no desenvolvimento emocional e cognitivo posterior (Oppenhein et al., 1988). Principalmente, diferenças qualitativas na responsividade, atitudes e características de personalidade maternas demonstraram-se estar associadas à qualidade do desenvolvimento adaptativo da criança.

Recentemente, autores como Schaffer (1996), Kochanska (1997) e Schermann et. al.(1997) retomaram essas questões, formulando os pressupostos para a adoção do modelo ao qual chamaram de competência social. Mediante esse modelo interações precoces satisfatórias entre mãe e criança estariam mediadas por um sentimento mútuo de eficácia. Baseada nessa mutualidade a criança mostra-se apta a prover experiências contingentes à mãe, evidenciando comportamentos preditivos e sendo responsiva. Numa situação de sincronia, a mãe interpreta os sinais da criança e intervém sensitivamente, gerando um ciclo de interações produtivas.

A habilidade materna em perceber, interpretar e responder às necessidades comunicativas da criança adquire posição central na construção de uma relação de mutualidade (Schaffer, 1996). Para isso muito contribuem as respostas seletivas dos bebês e seu potencial de atenção, pronto para a fixação do olhar em padrões semelhantes ao da face humana, forma e tamanho (Slater, 1997) e seus padrões de reatividade próprios (Brazelton, 1973).

O contato olho a olho é considerado como uma das redes de comunicação mais potentes, sendo um liberador inato de respostas maternais e potente facilitador de interação. A sinalização de estados pelo bebê (choro, sorriso, vocalização) promove elos na cadeira interativa quando a mãe se prontifica a atendê-los com satisfação.

Tais fatos verificados no cotidiano das relações mãefilho apontam que os vários sistemas senso-motores e comunicativos do bebê, a sensibilidade dos bebês a contrates e à discriminação de estímulos cromáticos e acromáticos. são facilitadores de um modelo diádico de interação, onde os com- portamentos de cada membro da díade complementam-se mutuamente.

Segundo Schermann et al. (1997) o padrão de reciprocidade presente na relação mãe-criança, pode ser mensurado pelo sentimento mútuo de eficácia, ou seja, o quanto cada membro da díade provê ao outro experiências apropriadas. A intensificação ou decréscimo dessas condições interativas dependerá dos padrões característicos de cada par mãe-bebê.

Lyra (1992; 1994) e Lyra et al. (1996), analisando o emergir do comportamento comunicativo bebê-mãe do ponto de vista de sua construção diádica, enfocou nessa relação: a produção de sons semelhantes a vogais, nas interações face-a-face e o movimento de extensão e posterior abreviação dessas interações em treze pares mães-crianças, os quais foram observados semanalmente pela técnica de videotape. A autora descreveu minuciosamente o engajamento em dois tipos de brincadeiras referenciais - tocar o lábio da mãe em contato facea-face e manipular um brinquedo conjuntamente, no chão.

Foram registrados de uma maneira dinâmica, a proximidade de contato do olhar e interrupções desses movimentos, que ocorreram nos encontros diádicos. Lyra, Cabral e Pantoja (1996), em outro artigo, salientaram os aspectos interativos na construção social objeto-mãe-bebê. Dos três estudos realizados, concluem-se que muitas práticas de rotina ocorrem acompanhadas de inovação, sendo que condutas emocionais, sorrisos e vocalizações acompanham usualmente esse tipo de interação. As autoras sugerem que as mudanças desenvolvimentais emergem não somente em extensão, mas dentro da referência de cada nova ação, bem como as rotinas e bases emocionais parecem servir de apoio à condução da inovação na interação.

Num simpósio realizado em parceria com Lyra (1994), Fogel, Holt e Dedo (1994) focalizaram no seu artigo os processos observados nas mudanças desenvolvimentais em díades mãe-infantes. À despeito do modelo parental como socializador da criança, os dados oriundos da pesquisa sugerem que muito do que acontece nessa relação é melhor compreendido como emergindo de mútuas co- regulações nas quais ambos os parceiros contribuem ativamente. Onze pares mães-bebês foram observados por vídeo no primeiro ano de vida, semanalmente e no segundo ano, a cada duas semanas. $\mathrm{O}$ artigo descreve as mudanças observadas entre 9 e 18 meses, em dois tipos de contextos de brincadeiras. A mãe iniciava cada um dos jogos; a criança segui-a por algum tempo e a seguir havia a dissolução da brincadeira. Os resultados demonstram que há mudança no formato, conteúdo e tempo em que as brincadeiras se desenvolvem, sofrendo muitas inovações em sua decorrência. Tanto a situação de difusão quanto de inovação foram compartilhadas nos formatos de jogos consensuais. Registros sobre lembranças de como iniciar, mas, principalmente, a iniciativa de criar sustentaram os episódios em que mães e bebês se envolveram, negociando formas de atuação.

Apesar de que na década de 90 iniciaram-se muitos estudos processuais, registrando-se em vídeo essas interações há também todo um acervo de pesquisas geradas tomando 
por referência características proeminentes de crianças e de suas mães que influenciam essas relações.

Nessa revisão, em primeiro lugar, procurar-se-á destacar as pesquisas que salientaram a estabilidade de características infantis e seus reflexos no comportamento da mãe.

\section{Estabilidade em características infantis}

Dados de pesquisas sobre os comportamentos dos neonatos os caracterizam como organismos complexos, com padrões de reatividade e de preferências próprios, desde o nascimento. Tem sido demonstrado que tais características afetam os comportamentos das pessoas que convivem com eles, sugerindo que ações e estilos de comportamento exibidos pelo infante desempenham papel importante no tipo e quantidade de estimulação que ele poderá receber desses agentes.

Nesses estudos, um número amplo de características tem sido relatado, como os estados, a responsividade à estimulação, as características físicas. Termos, como tendências, reatividade, taxas de responsividade diferencial, características organísmicas, temperamento e estilos de interação têm sido arrolados para designar a ocorrência de tais aspectos.

Estudos conduzidos acerca da estabilidade de características infantis preocuparam-se, primariamente, com o temperamento, comportamento interativo social e competência desenvolvimental do infante. Dentro dessa primeira área de pesquisa, foram investigados: a) características temperamentais infantis, como tendência à reatividade, estilos de organização, características organísmicas, estados predominantes, nível de atividade e humor as quais mostram uma forte estabilidade, ao longo do processo de desenvolvimento; b) observou-se, ainda, que estas características expressas sob as formas de: alerta visual, choro e nível geral de atividade se mantêm relativamente constantes, independentemente do grau e tipo de estimulação (Brazelton, 1973, Kaye \& Charney, 1980).

Grande número de pesquisadores tem sugerido que diferenças individuais entre infantes estão presentes, já ao nascimento, e, como tal, afetam características maternas e a experiência infantil em cada etapa de seu desenvolvimento posterior. Para investigadores interessados na avaliação de diferenças individuais em crianças, o temperamento tem sido um construto instigador.

Pettit e Bates (1984) estudaram extensivamente os fatores subjetivos na percepção do temperamento das crianças, demonstrando que numerosas características paternas influenciam os valores dos pais em relação aos filhos, incluindo o sexo dos pais, etnia, status sócio-econômico, estado de saúde mental e extroversão (Zeanah, Keener \& Anders, 1986, citado por Wolk, Zeanah, Coll \& Carr, 1992).

Além disso, pais que possuam conceitos negativos sobre si mesmos tendem a avaliar mais negativamente o temperamento dos filhos; pais que apresentam altos níveis de ansiedade e depressão também avaliam o temperamento de seus filhos como sendo mais difícil (Zeanah, Keener \& Anders, 1986, citado por Wolk et al., 1992).

Essas pesquisas são esclarecedoras das influências paternas, especialmente da tendência dos pais em desenvolve- rem uma representação social que, em certo grau resiste às mudanças ao longo do tempo.

Escalas do tipo Likert, contendo itens, com versão para mães e para pais foram aplicadas nessas pesquisas. A escala determina sentimentos afiliativos e interações avaliadas dos pais com relação a seus filhos. Quatro fatores do temperamento infantil têm sido registrados nessas investigações: Inquietude/Dificuldade - refere-se à apreciação dos pais do montante de inquietude e choro de seus filhos; Inadaptação - refere-se à apreciação pelos pais da dificuldade de ajustamento de suas crianças às mudanças na rotina; Imprevisibilidade - refere-se aos relatos dos pais sobre suas dificuldades em predizer a regularidade do comportamento de suas crianças e Disponibilidade - refere-se à combinação do nível de atividade das crianças e a intensidade das expressões emocionais positivas das mesmas.

A avaliação do temperamento como um traço estável ${ }^{1}$ foi feita mediante outra escala: a SITA, que registra o grau de Atividade Infantil. Ela compõe-se de duas partes: a primeira inclui as apreciações globais dos pais (numa escala Likert de 7 pontos) acerca do temperamento infantil, abrangendo: nível de atividade, ritmo, regularidade, adaptabilidade, flexibilidade, intensidade das expressões emocionais, especialmente a positividade do humor, comunicabilidade, irritabilidade, e sociabilidade. A segunda parte abrange 13 itens a respeito do nível de atividade da criança em situações rotineiras. A soma desses itens compreende uma medida única do nível de atividade e características do bebê.

Em laboratório, procedimentos experimentais fazem a apresentação de uma série de estímulos gradualmente estressantes, iniciando com cuidados de rotina e estimulação social, aumentando para estímulos estressantes mais complexos. Os estímulos são apresentados numa ordem de modo a requisitar, gradualmente, um número maior de modalidades reativas e sensoriais envolvidas dos nenês. $\mathrm{O}$ videotape registra nessas situações, os movimentos motores globais (sobressaltos e movimentos de pernas, braços e cabeça) e movimentos motores refinados (movimento das mãos; dos pés e dedos dos bebês) aos estímulos estressores. Além disso, cada vez que uma criança chora, durante a sessão, as intervenções comunicativas requeridas para acalmar a criança são avaliadas e categorizadas nos itens (1) quietude da criança; (2) apenas o examinador fala; (3) examinador fala e toca a criança; (4) pega a criança; (5) a mãe intervém.

A convergência entre o comportamento infantil na avaliação laboratorial e percepções resultantes de observações pelos pais demonstraram-se, em geral, bastante concordantes.

\section{Estados e reatividade geral}

Estado é normalmente considerado como um continuum de comportamento que reflete alguma condição orgânica subjacente, responsável por limiares de responsividade ou de níveis de consciência (Csilag, 1992; Lewis, 1972).

Diferenças de estados, de criança para criança, têm sido associadas à história constitucional biológica e pessoal, muito embora possa se supor que o meio exerça algum efeito 
sobre tais condições. A modulação de estados provê um índice do controle que o infante exerce sobre seu próprio comportamento; em adição, os estados influenciam o nível de adaptação dos infantes ao seu ambiente (Brazelton \& Als, 1986).

Diferenças congênitas respondem pelo tônus muscular, motilidade, vigor da sucção, estabilidade nos ciclos de sono e alerta e frequiência do sorriso. Assim, em relação aos estados predominantes do bebê, diferenças individuais em reatividade autonômica (taxas de responsividade diferencial - as quais envolvem tonicidade muscular e motilidade geral, vigor de sucção e outras respostas, conforme Horowitz $(1978$; 1980) desempenham papel importante na mediação de experiências maternas e afetam a interação na relação criança- agente cuidador.

Korner (1974) sintetizou nove dessas variáveis que caracterizam individualidade do infante, estudando-as em seus efeitos sobre os pais: (1) freqüência e duração dos períodos de inatividade e de alerta; (2) multisensorialidade ou especificidade de reação à estimulação externa; (3) estados e reatividade geral; (4) respostas a padrões de estimulação múltiplos ou competitivos; (5) diferenciação de estados; (6) opção por um canal de alívio; (7) modo de expressão desse canal; (8) diferenciação do comportamento e (9) predizibilidade. Tais reações diferenciam crianças quanto a padrões de choro, predisposição à interação, manutenção de contato visual e corporal, especialmente relevante no caso de autismo ou vulnerabilidade física ou mental.

Os períodos de alerta visual são extremamente importantes, particularmente nos meses iniciais, dada à imobilidade relativa do nenê e de amplos períodos de sono.

Becker e Thoman (1983) observaram que infantes que despendam comportamento de alerta por grandes períodos demonstraram-se mais capazes de orientação visual do que outros. A quantidade de responsividade visual é dependente da quantidade de tempo despendida em estado de alerta.

Outros autores enfatizam a importância do estado de alerta do infante para a necessária interação ambiental. Apontam que a manutenção de tal estado dependente da habilidade da mãe para eliciá-lo e de normas e padrões culturais que o estimulem; sugerem que tal procedimento é eminentemente cultural. Por exemplo, o contato olho-a-olho, na relação, é uma condição de eliciação maternal que estimula um estado proprioceptivo de alerta no bebê, bem como orienta a busca de outros padrões de estímulos efetivos à exploração.

Em bebês recém-nascidos, muito do que ocorre na interação está relacionado aos estados do infante e às percepções das mães como competentes ou experientes para tratá-los. A cada estado receptivo ou organizado do infante, há uma reação paralela de intervenção maternal. A discriminação de um estado de alerta no bebê provê um nível diferenciado de experiência e de competência à mãe. Sentimentos avaliáveis de competência dos agentes de cuidados podem, presumivelmente, estar relacionados a essas variáveis (Brazelton, 1973).

As descobertas dessas pesquisas podem ser resumidas como seguem: (1) temperamento infantil - como relatado pelos pais é moderadamente constante no período dos dois primeiros anos; (2) medidas objetivas de comportamento interativo social infantil, tais como sorrir, olhar, vocalizar à mãe, não têm geralmente mostrado estabilidade além dos primeiros dois anos (Clarke-Stewart, 1986); (3) competência desenvolvimental infantil, medida por testes padronizados, é moderadamente estável além dos primeiros dois anos mas não prediz o desempenho cognitivo posterior em nenhuma proporção (veja revisão de McCall, 1984).

\section{Diferenças de reatividade relativas a gênero}

Moore (citado por Horowitz, 1987) indicou diferenças na orientação e sensibilidade a tipos especiais de estimulação (visual, auditiva) entre os sexos, em infantes entre 9 e 14 semanas. Tem sido reconhecido, a partir das muitas pesquisas realizadas nessa área, que a habilidade para reforçar ou suprimir os balbucios emitidos por bebês é um importante evento ambiental que afeta significativamente o desempenho verbal em formação.

Os dados sobre comportamentos simultâneos indicam que há uma correlação relativa entre comportamento da mãe e da criança. Por exemplo, mães que vocalizam e sorriem mais para seus filhos também têm filhos que vocalizam e sorriem mais. $\mathrm{O}$ comportamento de choro da criança está associado à vocalização da mãe, ao pegar, ao alimentar, trocar ou sorrir. Vocalização e olhar da criança estão associados a tocar, acariciar, vocalizar, brincar e sorrir, pela mãe. Choro, movimentarse e brincar da criança foram, na maior parte, eliciadores dos comportamentos maternos. Vocalizações e sorrisos da criança são, na maior parte, eliciados pela mãe. O desenvolvimento da entonação vocal e o provimento de oportunidades e reforço contingente à produção de balbucio tem demonstrado afetar a frequiência e tipo de vocalização (F. D. Horowitz, comunicação pessoal, outubro de1992).

Da mesma maneira como ocorre em relação aos estados, a forma como a mãe responde às vocalizações dos infantes é extremamente importante, sugerindo que, face a isso, as meninas obteriam mais reforço diferencial por suas vocalizações e também eliciariam mais respostas verbais. Em conseqüência, avançariam na área de linguagem mais tarde e com mais facilidade, em comparação aos meninos.

Embora alguns dados sugiram essas relações, os achados das pesquisas nessa área não são totalmente consistentes.

\section{A idade e o nível de desenvolvimento da criança como fatores interativos}

No aspecto relativo à interação social, alguns estudos procuraram elucidar o significado das respostas dos pais sobre infantes de diferentes idades.

Uma das condições importantes que ocorrem nas atitudes das mães e que parece ser função da idade do infante diz respeito a mudanças nas formas de contato proximal e distal com as crianças, ao longo do desenvolvimento (Baydar \& Brooks-Gunn, 1991).

O conceito de responsividade maternal como empregado usualmente na literatura inclui várias formas de comportamento sobre uma variedade de sinais infantis: a vocalização está incluída a outros comportamentos maternos e infantis. 
As pesquisas indicam que um certo número de fatores influencia as mudanças processadas no desenvolvimento infantil, como: a hiperatividade, a mobilidade e curiosidade aspectos que intensificam a exploração do ambiente pela criança e induzem a mudanças na orientação e padrão de atenção maternos. Nessas novas situações, as mães tendem a substituir as formas mais próximas de controle (reforços e punições contingentes) por um sistema de sinais e de regras que passam a regular o comportamento à distância. Esse fator interativo responde por um incremento significativo no desenvolvimento cognitivo do infante.

Vygotsky (1984) sugeriu que a "internalização dessas regras", que orienta a comunicação entre mãe e criança constitua a base primária do desenvolvimento de práticas sociais junto ao infante.

\section{Tipos de interação e características maternas}

No processo interativo, as características maternas têm mostrado marcas mais eminentes de estabilidade do que as características infantis (Ainsworth, Blehar, Walters \& Wall, 1978).

Duas classes gerais de comportamento materno têm sido examinadas $: 1^{\circ}$ ) cuidados a necessidades físicas de rotina, e não envolvimento; e $2^{\circ}$ ) responsividade e interação voltada à diversão ou brincadeiras e estimulação educacional. Os cuidados de rotina não são estáveis (Clarke-Stewart, 1986) e não predizem a competência subseqüente, mas o envolvimento com estimulação vocal orientada ao objeto é amplamente estável e está diretamente correlacionado com resultados subseqüentes de competência infantil (Bradley, Caldwell \& Stedman, 1977; Zamberlan, Grossi, Moura \& Boldo, 1995, utilizando o Inventário Home, na descrição de interações mães com crianças pré-escolares).

\section{Atitudes maternas}

Brazelton (1973) observou que mães apresentam diferentes atitudes em relação a seus infantes normais e com problemas no desenvolvimento - "infantes de risco".

Além de Brazelton, outros investigadores, como Thoman (1979) demonstraram que a interação inicial mãe-criança facilita ou interfere no desenvolvimento adaptativo do infante, sendo que vários aspectos desse desenvolvimento são indicadores de conseqüências atuais e posteriores.

Outros autores (Fleming, Ruble, Flett \& Shaul, 1988) salientam como algumas condições e características maternas, tais como: insensibilidade, distúrbios psicológicos depressivos crônicos, ansiedade extrema, manifestos nas relações iniciais ou durante o curso da infância, afetam adversamente, o desenvolvimento infantil. Altos níveis de ansiedade materna, desinteresse maternal ou inconsistências nos cuidados, são extremamente relevantes ao status de desenvolvimento emocional, social e cognitivo dos infantes.

Outros estudos indicam que variações culturais e educacionais afetam a responsividade materna de maneiras específicas, confirmando e ampliando dados sobre as hipóteses de que a escolaridade maternal e o ambiente sócio-cultural emergem como influências importantes na responsividade materna.

Os determinantes e conseqüências da interação verbal prevalecem como tópicos importantes de investigação, sobretudo se o nível materno de responsividade, o qual é culturalmente afetado por convenções, afeta as relações de cuidados à criança.

A associação entre a educação formal materna e seu comportamento em relação às crianças pré-escolares é familiar na pesquisa sobre o desenvolvimento infantil. Mães de classe média alta e com educação a nível colegial ou mais, tendem a empregar um maior montante de interação verbal comparadas às mães de classe média e demonstraram ter crianças que desempenhavam melhor na linguagem aos dois anos. $\mathrm{O}$ montante de interação verbal que a criança recebe em idade precoce é importante e provavelmente relacionado ao nível educacional da mãe (Richman, Miller \& Levine, 1992).

Pesquisadores que postulam a implementação de programas especiais de estimulação, em geral qualificam no ambiente de crianças procedentes de famílias de baixa renda, estilos inadequados de interação familiar, baixa estimulação verbal/cognitiva, principalmente na interação materno-infantil de mães com pouco grau de instrução e trabalhadoras manuais, ou de suas substitutas.

Dados referentes a famílias de classe média, nos Estados Unidos (Tudge \& Putnam, 1997) em geral, demonstraram serem estas mais responsivas e abertas à idéia de atendimento às necessidades das crianças, sendo mais permissivas e democráticas nas formas de controle social de seus filhos. A diminuição de restrições e punições às crianças é também maior nessas famílias, principalmente a partir da década de 70. (citado por Frem, 1986).

Tais dados têm importância significativa em programas de intervenção junto a essas crianças e mães.

Zamberlan (1994) estudou vários aspectos do lar, considerados como relevantes para o desenvolvimento cognitivo, entre eles, destacando-se a interação verbal dos pais com as crianças, a posição da criança na família, a qualidade e variedade de brinquedos e estímulos disponíveis no ambiente. Tanto os últimos quanto os primeiros aspectos (e principalmente esses) demonstraram estar correlacionados com o desenvolvimento psico-social em uma comunidade de famílias e crianças em situação de risco.

\section{Conclusões}

Este trabalho se propôs a revisar as principais posições teóricas sobre a relação mãe-criança e possíveis decorrências metodológicas de tais concepções nos dados empíricos delas resultantes.

A análise de dados empíricos acerca das características dos bebês, embora apontem algumas demonstrações de estabilidade, indicam também que essas são estáveis apenas nos anos iniciais, não permitindo conclusões generalizáveis sobre a natureza de sua continuidade ao longo de todo o desenvolvimento infantil. 
Cumpre ressaltar também que essas pesquisas enfocaram, geralmente, e com prioridade, os aspectos de competência social, temperamental e afetivo da criança.

Supomos que generalizações desse tipo a outros domínios necessitam análise mais cuidadosa, visto que a pesquisa sobre multi-modalidades perceptivo-sensoriais no neonato (Mehler \& Fox, 1985) tem revelado a complexidade dos sistemas comportamentais dos infantes e suas influências na interação.

A esse respeito, lembramos Horowitz $(1978 ; 1980)$ que ressalta a importância de se avaliar tanto as dimensões universais como culturais nos processos de desenvolvimento e sugere que a identificação precoce de tais características pode proporcionar um avanço na compreensão do sistema de intercâmbios que a criança experiência no curso de sua vida.

Concluindo, destacamos que dada a importância dessas variáveis dos infantes quanto das relacionadas aos agentes com os quais as crianças interagem, acreditamos que seja necessário o aprofundamento dessas questões, via pesquisa, subsidiando a atuação de natureza preventiva à ocorrência de problemas na interação entre esses agentes, com possíveis consequiências para o processo de desenvolvimento.

\section{Referências}

Ainsworth, M. D., \& Wittig, B. A. (1969). Attachment and exploratory behavior of one- year-olds in a strange situation. Child Development, 31, $67-71$.

Ainsworth, M.D., Blehar, M.C., Walters, E., \& Wall, S. (1978). Patterns of attachment. Hillsdale: Erlbaum.

Bradley, R., Caldwell, B. M., \& Stedman, D. J. (Orgs.). (1977). Infant education: A guide for helping handicapped children in the first three years. New York: Walker.

Baumrind, D., \& Black, A. E. (1967). Socialization patterns associated with dimensions of competence in preschool boys and girls. Child Development, $38,291-327$.

Baydar, N., \& Brooks-Gunn, J. (1991). Effects of maternal employment and child-care arrangements on preschooler's cognitive and behavioral outcomes: Evidence from the children of the National Longitudinal Survey of Youth. Developmental Psychology, 27(6), 932-945.

Becker, P. T., \& Thoman, E. B. (1983). Organization of sleeping and waking states in infants: Consistency across contexts. Psychology \& Behavior, 31, $405-410$.

Biasoli-Alves, Z. M. M. (1992). Discutindo o desenvolvimento da criança e do adolescente. Manuscrito não-publicado.

Blurton Jones, N. (1983). (Org.). Estudos etológicos do comportamento da criança. São Paulo: Pioneira.

Bowlby, J. (1989). Uma base segura: implicações clínicas da teoria do apego. Porto Alegre: Artes Médicas.

Bowlby, J. (1991). Attachment and loss (vol. 1.) Harmondsworth: Penguin (Texto original publicado em 1951).

Brazelton, T. B. (1973). Neonatal Behavioral Assessment Scale. Philadelphia: Lippincott.

Brazelton, T. B., \& Als, H. (1986). Importance of early intervention. In J. P. Gomes (Org.), Biopsychology of parent-infant (cap. 2, pp. 15-30). Lisboa: Communication International Symposium, Fund. Calouste Gulbenkian.

Brody, G. H., Stoneman, Z., \& Burke, M. (1988). Child temperament and parental perceptions of individual child adjustment: An intrafamilial analysis. American Journal of Orthopychiatry, 58, 532-542.
Clarke-Stewart, K. A. (1986). Interactions between mothers and their young children: Characteristics and consequences. Monographs of the Society for Research in Child Development - Serial, no. 253, 58, 6-7.

Csilag, S. (1997). Os três primeiros dias de vida: uma observação dos estados comportamentais do bebê recém-nascido. Tese de Doutorado não-publicada, Universidade de São Paulo, São Paulo

Feinman, S., \& Lewis, M. (1988). Social referencing at ten months: A second order effect on infant's responses to strangers. Child Development, 54, 878887.

Fogel, A. (1988). Cyclicity and stability in mother-infant face-to-face interaction: A comment on Cohn and Tronick. Development Psychology, 24(3), 393395.

Fogel, A. (1992, outubro). Developing through relationships. Conferência proferida na XXIII Reunião de Psicologia da Sociedade Brasileira de Psicologia, Ribeirão Preto, SP.

Fogel, A., Holt, S. A., \& Dedo, I. Y. (1994). Innovation diffusion and dissolutions as process of development in mother-infant relationships. Paper 1 presented to Symposium. In A. Fogel (Org.), International Conference on Personal Relationships. Gronigen, Meeting in The Netherlands, July, $4-8$.

Fleming, A. S., Ruble, D. N., Flett, G., \& Shaul, D. L. (1988). Postpartum adjustment in first-time mothers: Relations between mood, maternal attitudes and mother infant interactions. Developmental Psychology, 24(1), 71 -81.

Freud, S. (1959). Os dois princípios do suceder psíquico. In Obras Completas (vol. 7, pp. 321-329). Rio de Janeiro: Delta.

Frem, M. H. S. (1986) A educação dos filhos menores na década de 70: a busca do melhor? Dissertação de mestrado não-publicada, Universidade de São Paulo, São Paulo.

Horner, T. M. (1988). Rapprochement in the psychic development of the toddler: A transactional perspective. American Journal of Orthopsychiatry, 58(1), 515.

Horowitz, F. D. (1978). Methods of assessment for high risk and handicapped infants. In C. Ramey \& P. Trohanis (Orgs.), Finding and educating the high-risk infant (pp. 171-206). New York: Mc Graw Hill.

Horowitz, F. D. (1980). Intervention and its effects on early development: What model of development is appropriate. Life-span development intervention. New York: Academic Press.

Horowitz, F. D. (1987). Exploring developmental theories: Toward a structural/ behavioral model of development. New Jersey: Erlbaum.

Kaye, R., \& Charney, R.(1980). How mothers maintain "dialogue" with twoyears-old. In D. Olson (Org.), The social foundations of language and thought (pp. 211-230). New York: Norton.

Kochanska, G. (1997). Mutually responsive orientation between mothers and their young children: implications for early socialization. Child Development, 68(1), 94-112.

Korner, A. F. (1974). The effect of the infant's state, level of arousal, Sex and ontogenic stage on the caregiver. In M. Lewis \& L. A. Rosenblun (Orgs), The effect of the infant on its caregiver (pp.187-214). New York: J. Wiley $\&$ Sons.

Lyra, M. C. D. P. (1992, outubro). Características do processo de construção social no início da vida. Comunicação apresentada na XXIII Reunião Anual da Sociedade Brasileira de Psicologia, Ribeirão Preto.

Lyra, M. C. D. P., \& Fogel, A. (1994). Innovation as historical linkage in social change processes. Paper 2 presented to Symposium. In A. Fogel (Org.), International Conference on Personal Relationships, Gronigen, Meeting in The Netherlands, July, 4-8.

Lyra, M. C. D. P. ( 1992). Características do processo de construção social no início da vida. Comunicação Oral apresentada na XXII Reunião Anual da Sociedade de Psicologia de Ribeirão Preto, Ribeirão Preto, S. P.

Lyra, M. C. D. P., Cabral, E. A., \& Pantoja, A. P. F. (1996). Construction of shared meaning in mother-object-infant dyad. In M. C. D. P. Lyra \& J. 
Valsiner (Orgs.), Child development in culturally structured environments (Vol. 4). Norwood: Ablex.

Lyra, M. C. D. P., \& Valsiner, J. (1996). Introduction: Communication as a construction process. In M. C. D. P. Lyra \& J. Valsiner (Orgs.), Construction of psychological processes in course of interpersonal communication (Vol. 4, pp. 1-4). Norwood: Ablex.

McCall, R. B. (1984). Developmental changes in mental performance: The effect of the birth of a sibling. Child Development, 55, 1317-1321.

Mehler, J., \& Fox, R. (1985). (Orgs.). Neonate cognition beyond the blooming buzzing confusion. New Jersey: Lawrence Erbaum.

Oppenhein, D., Sagi, A., \& Lamb, M. E. (1988). Infant-adult attachments on the kibbutz and their relation to socioemocional development 4 years late. Developmental Psychology, 24(3), 427-433.

Osofsky, J. D., \& Connors, K. (1979). Mother-infant interaction: An integrative view of a complex system. In J. D. Osofsky (Org.), Handbook on Infant Development (pp. 462-518). New York: J. Wiley \& Sons.

Pettit, G. S., \& Bates, J. E. (1984). Continuity of individual differences in the mother-infant relationship from six to thirteen months. Child Development, $55,729-739$

Power, T. G., \& Chapiesky, M. L. (1986). Child rearing and impulse control in toddlers: A naturalistic investigation. Developmental Psychology, 22, 271-275.

Pratt, M. W, Cowan, P. A., Kerig, P., \& Cowan, C. P. (1988). Mothers and fathers teaching 3 year-olds: Authoritative parenting and adult scaffolding of young children's learning. Developmental Psychology, 24(6), 832-839.

Richman, A. L., Miller, P., \& Levine, R. A. (1992). Cultural and educational variations in maternal responsiveness. Developmental Psychology, 28(4), 614-621.
Rossetti-Ferreira, M. C. (1986). Mãe-criança: separação e reencontro. São Paulo, Edicon.

Schaffer, H. R. (1996). Social development. Oxford: Blackwell.

Slater, A. (1997). Visual perception and its organization in early infancy. In G. Bremner, A. Slater \& C. Butterworth (Orgs.), Infant development: Recent advances (pp. 34-37). New Jersey: Erlbaum.

Stedman, M. E. (1991). Skinner's utopia: Bi-directional influences. Psychological Reports, 69, 722 .

Thoman. E. B. (Org.). (1979). The origins of the infant's social responsiveness. New York: John Wiley \& Sons.

Tudge, J., \& Putnam, S. E. (1997). The everyday experiences of North American preschoolers in two cultural communities: A cross-disciplinary and crosslevel analysis. In J. Tudge, M. J. Shanahan \& J. Valsiner (Orgs.), Comparisons in human development: Understanding time and context (pp. 252-281). New York: Cambridge University Press.

Vygotsky, L. S. (1984). A formação social da mente. São Paulo: Martins Fontes. Wolk, S., Zeanah, C. H., Coll, C. T. G., \& Carr, S. (1992). Factors affecting parent's perceptions of temperament in early infancy. American Journal of Orthopychiatry, 62(1), 71-82.

Zamberlan, M. A. T. (1994). Ambientes de desenvolvimento de crianças préescolares: proposta de intervenção preventiva familiar para crianças em situação de risco. Relatório de Pesquisa Pós-doutorado, USP, Ribeirão Preto, S. P., Bolsa CNPq. Processo n ${ }^{\circ} 150.012 / 94$.

Zamberlan, M. A. T, Grossi, R., Moura, C. B., \& Boldo, M. A. (1995). Riscos ambientais à criança: reflexões para uma proposta de intervenção preventiva junto à família. Torre de Babel: Reflexões e Pesquisas em Psicologia, 2(1), 7-29.

Nota

1 Essas modalidades de pesquisas, iniciadas por Pettit e Bates (1984) foram refinadas nas proposições e instrumentos criados por Brody, Stoneman e Burke (1988) e passaram a constituir-se em parâmetros para estudos de tal natureza.

Maria Aparecida Trevisan Zamberlan, doutora em Psicologia Escolar pela Universidade de São Paulo, SP, Pós-doutorado em Psicologia do Desenvolvimento pela Universidade de São Paulo, Ribeirão Preto, SP, é professora do Departamento de Educação da Universidade Estadual de Londrina, PR.

Endereço para correspondência: Universidade Estadual de Londrina, Depto. de Educação, Programa de Mestrado em Educação. Avenida Celso Garcia Cid, s/nº Campus Universitário, 86.050-901, Londrina, PR. Telefone (21)43371.4338, Fax: (21)43371.4076. E-mail: zamba@ldnet.com.br. 